\section{Perspectivas de eliminação da hanseníase como problema de saúde pública no Estado do Paraná, Brasil}

\author{
Prospects for the elimination of leprosy as a public \\ health problem in the State of Paraná, Brazil
}

\begin{abstract}
Leprosy, an endemic contagious/infectious disease, is still a public health problem in most States of Brazil, despite an important decrease in the prevalence rate in recent years. The current study aims to analyze the prospects for the elimination of leprosy in the State of Paraná, based on detection and prevalence rates for the years 2000 to 2005, in addition to data on gender, age, clinical form, operational classification, and number of skin lesions. Data were obtained from the Reportable Diseases Information System (SINAN) and the Tabnet software. Leprosy detection and prevalence rates in Paraná remained unaltered during the study period. The leprosy detection rates were high or very high in the majority of the health districts in the State. In terms of prevalence, eight health districts had reached the goal of eliminating the disease. In conclusion, the State of Paraná is close to reaching the goal of eliminating leprosy. Based on the study results, actions are recommended to eliminate the disease as a public health problem.
\end{abstract}

Leprosy; Disease Notification; Prevalence
Reinaldo Antonio da Silva Sobrinho 1 Thaís Aidar de Freitas Mathias 2

\section{Introdução}

A estratégia adotada pelos países membros da Organização Mundial da Saúde (OMS) definiu, em 1991, que a hanseníase deixaria de ser um problema de saúde pública naqueles países onde o coeficiente de prevalência fosse menor ou igual a 1 caso para cada 10 mil habitantes $1,2,3$. No entanto, a hanseníase ainda não foi eliminada em Angola, Brasil, República Africana Central, República Democrática do Congo, Índia, Madagascar, Moçambique, Nepal e na República Unida da Tanzânia 2 .

O indicador utilizado atualmente para monitorar a eliminação da hanseníase é a prevalência pontual 4. A Portaria GM/MS $n^{o} .31$ 5, de 8 julho de 2005, estabeleceu que, para a construção do coeficiente de prevalência pontual, o numerador seja composto pelo total de pacientes em curso de tratamento, que representa o total de doentes no momento da análise. A prevalência pontual, usualmente, é medida no dia 31 de dezembro de cada ano. Até 2004, o indicador utilizado oficialmente era o coeficiente de prevalência que era composto pelos casos em curso de tratamento e incluía também os casos em abandono de tratamento, o que impossibilitava qualquer comparação da prevalência do Brasil com outros países que já adotavam tal método. Até então, o Brasil era o país com maior magnitude da hanseníase do mundo 5 . 
No início de 2005, a prevalência mundial da hanseníase era de 286.063 casos, e o número de casos novos descobertos, durante o ano de 2004, foi de 407.791. O número de casos descobertos, em todo o mundo, reduziu em torno de $107 \mathrm{mil}$ casos, isto é, $21 \%$ durante o ano de 2004, quando comparado com o ano de 2003. Essa redução ocorreu principalmente pela diminuição de casos novos na Índia, onde houve diminuição, na detecção, de $29 \%$ em relação ao ano de 20032 . Em 1998, a Índia foi responsável por 79\% dos casos de hanseníase detectados globalmente 6 . O número de casos novos tem diminuído na região oriental do mediterrâneo, na Ásia e regiões do Pacífico Ocidental. Porém, não há igual tendência na África e nas Américas 6.

A situação epidemiológica da hanseníase no Brasil é considerada heterogênea devido à grande variação do coeficiente de prevalência nas várias regiões do país. Em 2005, a Região Norte foi a que apresentou o maior coeficiente, 4,02 casos por 10 mil habitantes, seguida da Região Centro-Oeste, com 3,29 casos por 10 mil habitantes. Já o Nordeste apresentou 2,14 casos por $10 \mathrm{mil}$ habitantes; o Sudeste 0,60, caso; e o Sul, 0,53 caso por 10 mil habitantes 7 . Os principais indicadores de monitoramento de eliminação da hanseníase apontam para uma melhor situação da Região Sul em relação à Região Norte 8 , contudo, os coeficientes de detecção continuam elevados no Brasil e América Latina 9 .

Os estados do Rio Grande do Sul e Santa Catarina já eliminaram a hanseníase como problema de saúde pública, por apresentarem coeficientes de prevalência de 0,14 e 0,20 caso por 10 mil habitantes, respectivamente. No Estado do Paraná, o coeficiente de prevalência, em 1994, era de 15 casos por 10 mil habitantes; em 1999, de 3,3; em 2004, foi de 1,4; e, em 2005, de 1,12 caso por 10 mil habitantes 7,10, o que indica declínio sensível da endemia. Entretanto, esses números ainda são superiores à meta estipulada pelo Ministério da Saúde, mas a Secretaria de Saúde do Estado do Paraná manteve o compromisso de eliminar a hanseníase do estado até o ano de 2010, ou seja, alcançar o coeficiente de prevalência de 1 ou menos de 1 caso para cada 10 mil habitantes 10 , esforço que foi implementado em todos os países endêmicos 11 .

A significativa redução informada na prevalência registrada no Brasil é resultante também da atualização de registros dos pacientes e da unificação nas definições de um caso de hanseníase ${ }^{2}$. Com isso, vem ocorrendo uma aproximação do coeficiente de prevalência com a meta de eliminação. Na década de 90, era comum a continuidade do paciente em registro ativo mesmo após o término do tratamento, e, em situações de abandono ou óbito, esses casos que não recebiam alta no sistema de informação contribuíam para a manutenção do coeficiente de prevalência elevado.

Por intermédio do Plano Nacional de Eliminação da Hanseníase, os estados e municípios promoveram ações como a alta estatística e limpeza do banco de dados, dessa forma, ficaram registrados apenas os casos de hanseníase que realmente estavam em curso de tratamento. Atualmente, para atualizar os registros de hanseníase, foi elaborado, pelo Ministério da Saúde, um aplicativo específico para essa finalidade, o programa de computador HansWin (Centro de Vigilância Epidemiológica "Prof. Alexandre Vranjac”, São Paulo, Brasil), que viabiliza a leitura dos dados de modo a detectar inconsistências e identificar aqueles casos em abandono ou que ultrapassaram o tempo de tratamento previsto. Ao mesmo tempo em que os coeficientes de prevalência vêm diminuindo, os coeficientes de detecção, que indicam a endemicidade, continuam elevados em virtude da continuidade na transmissão da doença entre as populações ou ainda pela detecção tardia de casos.

No estudo realizado, na Índia, no período de 1982 a 2002, observou-se que o coeficiente de prevalência da hanseníase apresentou forte declínio, e o coeficiente de detecção continuou aumentando quase que constantemente, ultrapassando o coeficiente de prevalência desde 199912 . No Brasil, Martelli et al. 13 verificaram a mesma tendência no período de 1983 a 2000, no qual ocorreu forte declínio do coeficiente de prevalência da doença, ao passo que os coeficientes de detecção continuaram elevados e sem alteração. Conforme o estudo de Meima et al. 6, na América Latina, houve aumento consistente do coeficiente de detecção da hanseníase.

Ao longo do tempo, o Programa de Controle da Hanseníase no Estado do Paraná desenvolveu estratégias e ações conforme aquelas recomendadas pela OMS e pelo Ministério da Saúde. As atividades, como o diagnóstico, o tratamento, a avaliação de incapacidade física e o exame de contatos, eram realizadas apenas por dermatologistas nos centros de referência da rede pública de saúde. Em 1996, com a Norma Operacional Básica (NOB-96), iniciou-se, no Estado do Paraná, a estratégia de descentralização do atendimento e tratamento da doença para os municípios, e as informações sobre sinais e sintomas da doença, o exame dos contatos domiciliares dos casos novos continuam sendo privilegiados como estratégia no diagnóstico precoce da hanseníase.

No Estado do Paraná, entre as ações de eliminação da hanseníase, foi prioridade a capacitação dos profissionais da rede de atenção básica 
de saúde visando à descentralização e à responsabilização dos gestores e equipes municipais. No esforço final para atingir a meta de eliminação até o ano de 2010, a Secretaria de Estado de Saúde do Paraná comprometeu-se em continuar executando as ações do Plano Nacional de Eliminação da Hanseníase 10.

Tendo em vista que, no Estado do Paraná, o coeficiente de prevalência continua acima do preconizado pela OMS e pelo Ministério da Saúde e considerando a importância da eliminação da hanseníase como problema de saúde pública, foi objetivo deste estudo avaliar a perspectiva de eliminação da hanseníase no estado.

\section{Material e método}

Trata-se de um estudo descritivo exploratório em que foram estudados todos os casos novos e antigos de hanseníase em tratamento nos anos de 2000 a 2005, no Estado do Paraná, segundo Regionais de Saúde.

O Estado do Paraná é dividido administrativamente em 22 Regionais de Saúde, com população estimada, para 2005, em 10.261.840 habitantes. Conta com 399 municípios, dos quais, $80 \%$ têm menos de 20 mil habitantes e abrangem $28 \%$ do total da população do estado 10 . As Regionais de Saúde constituem-se como instâncias administrativas intermediárias da Secretaria de Estado da Saúde e do Instituto de Saúde e Epidemiologia do Paraná. Às Regionais de Saúde cabe desenvolver estratégias necessárias para apoiar os municípios em todas as áreas e para influenciar a gestão das questões regionais, fomentando a busca contínua e crescente da eficiência, como também direcionar o planejamento para ações em saúde de cada município em sua região 10 .

Foi utilizado o banco de dados do Sistema de Informação de Agravos de Notificação (SINAN), cedido pela Secretaria Estadual de Saúde do Paraná, para realizar o cálculo do coeficiente de detecção geral, coeficiente de detecção em menores de 15 anos, e, para fortalecer as discussões sobre o quadro endêmico da hanseníase no Estado do Paraná, foi feita também a análise descritiva das variáveis sexo, classificação operacional, classificação clínica e número de lesões de pele para o ano de 2005. Para a análise do coeficiente de prevalência pontual, o banco de dados usado foi Tabnet (Departamento de Informática do SUS, Ministério da Saúde, Brasil). Os dados populacionais, por faixa etária e ano, foram obtidos no sítio do Departamento de Informática do SUS (DATASUS) 14.

Para a Regional de Saúde de Ivaiporã, foi feito um ajuste no coeficiente de detecção referente ao ano de 2004, pois, nesse ano, houve erro de diagnóstico de casos da hanseníase em um município, elevando o coeficiente de detecção para 21,84 casos por 10 mil habitantes. Após o ajuste, o coeficiente de detecção dessa Regional de Saúde, para 2004, foi de 3,24 casos por 10 mil habitantes, construído por meio da média dos casos novos diagnosticados de 2000 a 2003 dividido pela população de 2004.

Os coeficientes de detecção geral e em menores de 15 anos foram analisados para o período de 2000 a 2005, e o de prevalência pontual da hanseníase para 2004 e 2005, segundo Regionais de Saúde.

Para a análise do coeficiente de prevalência pontual, foi consultada a base de dados de acompanhamento da hanseníase da página eletrônica do Ministério da Saúde (Tabnet). Esse programa permite a realização do cálculo da prevalência pontual da hanseníase utilizando o número de casos existentes em curso de tratamento conforme orientação da Portaria GM/MS no .31 5. Foram utilizados também os parâmetros de endemicidade para o coeficiente de detecção e de prevalência recomendados pelo Ministério da Saúde 14 e a meta de eliminação da hanseníase, que é atingir o coeficiente de prevalência de 1 ou menos de 1 caso para cada 10 mil habitantes 15 .

Os parâmetros de endemicidade para o coeficiente de detecção da hanseníase recomendados pelo Ministério da Saúde são os graus: $<0,20$, baixo; 0,20 -1, médio; 1 -2, alto; 2 -4, muito alto; $\geq 4$, hiperendêmico; e, para o coeficiente de detecção em menores de 15 anos, os parâmetros de endemicidade são: $<0,05$, baixo; 0,05 $\vdash 0,25$, médio; $0,25 \vdash 0,5$, alto; 0,5 -1,0, muito alto; > 1,0, hiperendêmico 15 . Para o coeficiente de prevalência: < 1, baixo; 1 -5, médio; 5 -10, alto; 10 -20, muito alto; $>20$, hiperendêmico 15 .

O presente estudo foi aprovado pelo Comitê de Ética e Pesquisa Envolvendo Seres Humanos da Universidade Estadual de Maringá, respeitando as normas estabelecidas na Resolução $n^{o}$. 196/96 do Conselho Nacional de Saúde (parecer no. 390/2005).

\section{Resultados}

O coeficiente de detecção da hanseníase para o Estado do Paraná foi de 1,62 e 1,60 caso por 10 mil habitantes, para os anos de 2000 e 2005, respectivamente, sendo que o ano de 2003 apresentou o maior coeficiente de detecção $(1,81$ caso por 10 mil habitantes) do período (Tabela 1). Em 14 Regionais de Saúde (Paranaguá, Irati, Pato Branco, Francisco Beltrão, Cascavel, Campo Mourão, Umuarama, Cianorte, Paranavaí, Apucarana, 
Cornélio Procópio, Toledo, Telêmaco Borba e Ivaiporã), o coeficiente de detecção foi maior no ano de 2005 quando comparado ao ano de 2000.

A Tabela 1 mostra que, em geral, as Regionais de Saúde de Foz do Iguaçu, Guarapuava, Cornélio Procópio e Ivaiporã apresentaram os maiores coeficientes de detecção do período, enquanto que as Regionais de Saúde de Paranaguá e Metropolitana apresentaram os menores. Nenhuma Regional de Saúde foi classificada com grau baixo de endemicidade. A maioria apresentou coeficiente de detecção alto e muito alto, e duas Regionais de Saúde foram classificadas como hiperendêmicas em alguns momentos do período (Foz do Iguaçu e Cornélio Procópio).

A prevalência pontual da hanseníase, no Paraná, nos anos de 2004 e 2005, foi de 1,20 e 1,12 caso por 10 mil habitantes (Tabela 1). A Regional de Saúde que obteve maior coeficiente de prevalência foi Ivaiporã, com 9,37 casos por 10 mil habitantes. Destacam-se também as Regionais de Saúde de Guarapuava e Foz do Iguaçu, com 3,01 e 2,68 casos por 10 mil habitantes, respectivamente (Tabela 1, Figura 1). Possivelmente o alto coeficiente de prevalência da Regional de Saúde de Ivaiporã esteja influenciado pelo erro de diagnóstico de casos ocorrido em 2004.

Constatou-se que as Regionais de Saúde permaneceram com o parâmetro de endemicidade médio (exceto a Regional de Saúde de Ivaiporã), e não houve Regional de Saúde classificada com coeficiente de prevalência alto, muito alto ou hiperendêmico no ano de 2005 (Tabela 1, Figura 1). O coeficiente de detecção, nos anos de 2004 a 2005, foi superior ao coeficiente de prevalência no Estado do Paraná.

Considerando os parâmetros de endemicidade do Ministério da Saúde para a detecção, a Figura 2 mostra (somente para o ano de 2005) que a maioria das Regionais de Saúde ainda permaneceu com grau de endemicidade alto (nove Regionais de Saúde) e muito alto (11 Regionais

Tabela 1

Coeficientes de detecção e de prevalência da hanseníase (por 10 mil habitantes) segundo Regionais de Saúde. Paraná, Brasil, 2000 a 2005

\begin{tabular}{|c|c|c|c|c|c|c|c|c|}
\hline \multirow[t]{2}{*}{ Regional } & \multirow{2}{*}{$\begin{array}{c}2000 \\
C D\end{array}$} & \multirow{2}{*}{$\begin{array}{l}2001 \\
C D\end{array}$} & \multirow{2}{*}{$\begin{array}{c}2002 \\
C D\end{array}$} & \multirow{2}{*}{$\begin{array}{c}2003 \\
C D\end{array}$} & \multicolumn{2}{|c|}{2004} & \multicolumn{2}{|c|}{2005} \\
\hline & & & & & $C D$ & $\mathrm{CP}$ & $C D$ & $\mathrm{CP}$ \\
\hline 1ạ Paranaguá & 0,68 & 0,69 & 0,40 & 0,39 & 1,01 & 0,53 & 0,73 & 0,80 \\
\hline 2a Metropolitana & 0,83 & 0,67 & 0,74 & 0,70 & 0,61 & 0,45 & 0,56 & 0,38 \\
\hline 3ạ Ponta Grossa & 1,71 & 1,21 & 1,85 & 1,55 & 1,42 & 1,00 & 1,36 & 0,90 \\
\hline 4a Irati & 0,73 & 0,93 & 0,92 & 1,05 & 0,77 & 0,58 & 1,48 & 0,77 \\
\hline 5a Guarapuava & 3,30 & 3,16 & 3,66 & 3,84 & 3,39 & 2,40 & 3,08 & 3,01 \\
\hline 6ạ União da Vitória & 1,60 & 1,46 & 1,57 & 0,99 & 0,79 & 0,92 & 1,21 & 0,60 \\
\hline 7ạ Pato Branco & 1,23 & 0,97 & 1,17 & 1,04 & 1,24 & 0,70 & 1,36 & 0,78 \\
\hline 8a Francisco Beltrão & 1,45 & 0,93 & 1,03 & 1,75 & 1,97 & 1,25 & 1,80 & 0,98 \\
\hline 9ạ Foz do Iguaçu & 3,26 & 3,33 & 4,68 & 4,46 & 2,72 & 2,68 & 3,07 & 2,45 \\
\hline 10ạ Cascavel & 2,25 & 2,21 & 2,90 & 2,76 & 2,39 & 1,71 & 2,63 & 2,37 \\
\hline 11ạ Campo Mourão & 2,48 & 2,13 & 2,30 & 2,27 & 3,01 & 2,36 & 2,74 & 1,93 \\
\hline 12a Umuarama & 1,78 & 1,68 & 1,46 & 2,32 & 2,01 & 1,01 & 2,36 & 1,08 \\
\hline 13ạ Cianorte & 1,75 & 2,46 & 2,05 & 2,35 & 2,25 & 1,56 & 2,94 & 1,62 \\
\hline 14ạ Paranavaí & 1,52 & 1,88 & 2,03 & 2,15 & 2,77 & 1,94 & 2,61 & 1,58 \\
\hline 15ạ Maringá & 1,16 & 1,36 & 1,65 & 1,55 & 1,00 & 0,63 & 1,16 & 0,79 \\
\hline 16a Apucarana & 1,18 & 1,58 & 1,59 & 2,08 & 1,64 & 1,29 & 1,72 & 1,14 \\
\hline 17ạ Londrina & 2,07 & 1,99 & 2,09 & 2,10 & 1,82 & 1,19 & 1,65 & 1,30 \\
\hline 18ạ Cornélio Procópio & 2,61 & 3,74 & 4,11 & 3,65 & 2,43 & 1,33 & 3,66 & 1,46 \\
\hline 19ạ Jacarezinho & 2,65 & 1,32 & 1,65 & 2,02 & 2,02 & 1,06 & 1,91 & 1,54 \\
\hline 20ạ Toledo & 1,80 & 2,32 & 2,60 & 2,78 & 2,69 & 1,51 & 2,13 & 1,39 \\
\hline 21a Telêmaco Borba & 1,75 & 1,74 & 1,98 & 3,70 & 2,68 & 1,16 & 2,97 & 1,76 \\
\hline 22a Ivaiporã & 2,79 & 3,13 & 3,32 & 3,11 & 3,24 & 9,37 & 3,91 & 2,14 \\
\hline Paraná & 1,62 & 1,55 & 1,78 & 1,81 & 1,54 & 1,20 & 1,60 & 1,12 \\
\hline
\end{tabular}

CD: coeficiente de detecção; CP: coeficiente de prevalência.

Fontes: Sistema de Informação de Agravos de Notificação (SINAN)/Secretaria Estadual de Saúde do Paraná, 2000 a 2005; Tabnet (Departamento de Informática do SUS, Ministério da Saúde, Brasil), 2004 e 2005. 


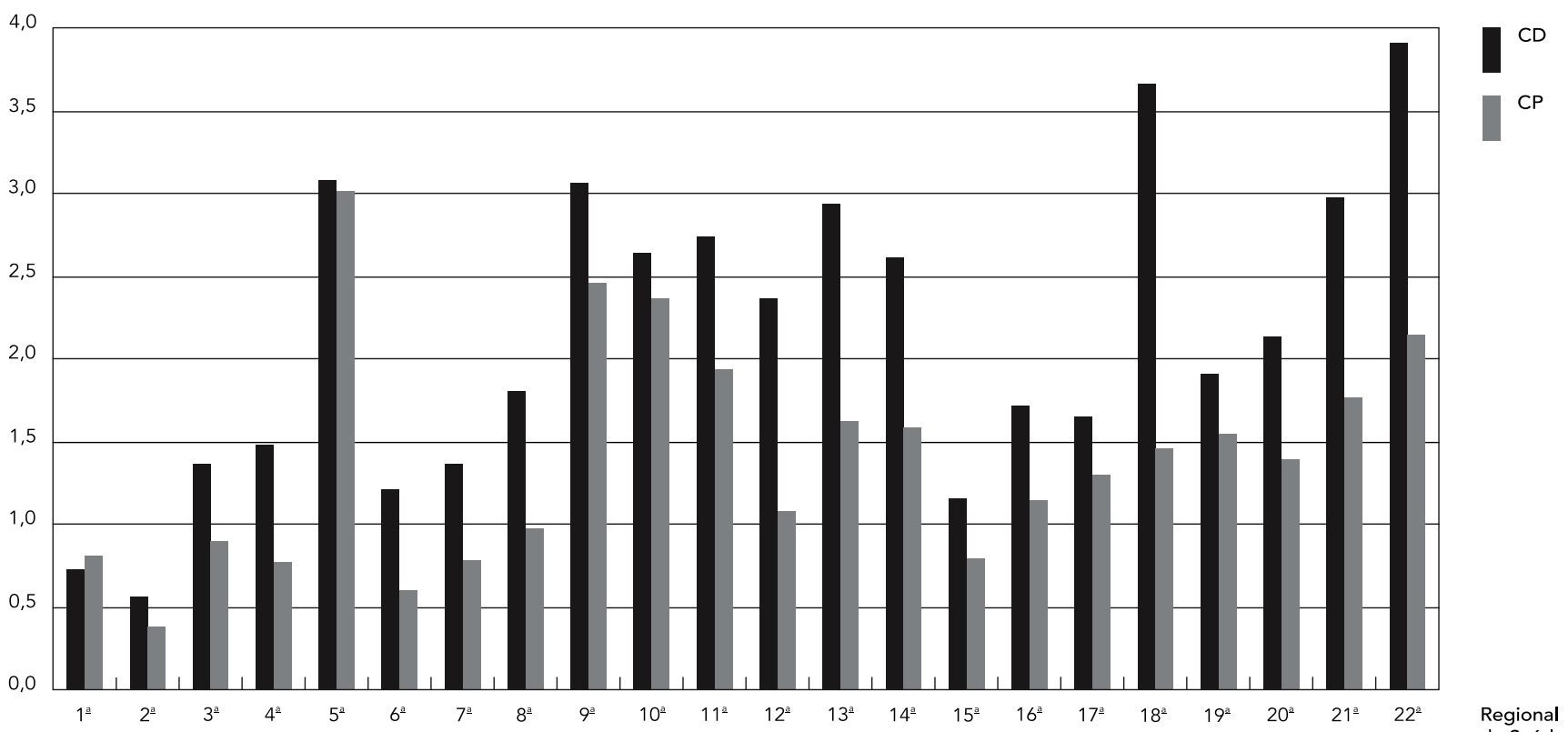

Fontes: Sistema de Informação de Agravos de Notificação (SINAN)/Secretaria Estadual de Saúde do Paraná, 2005; Tabnet (Departamento de Informática do SUS, Ministério da Saúde, Brasil), 2005.

de Saúde), ou seja, acima de 1 caso por $10 \mathrm{mil}$ habitantes. Apenas as Regionais de Saúde Metropolitana e Paranaguá apresentaram grau de endemicidade médio.

Observando os parâmetros de eliminação da hanseníase, na Figura 2, verifica-se que, no ano de 2005, oito Regionais de Saúde atingiram a meta de eliminação, qual seja, apresentarem coeficiente de prevalência menor que 1 caso por 10 mil habitantes. Por outro lado, existem Regionais de Saúde, como Guarapuava, Foz do Iguaçu, Cascavel e Ivaiporã, que ainda apresentaram coeficiente de prevalência que variam de 2,14 a 3,01 casos por 10 mil habitantes. Apenas a Regional de Saúde de Paranaguá apresentou, no ano de 2005, coeficiente de detecção inferior ao coeficiente de prevalência. Nas demais Regionais de Saúde, o coeficiente de detecção registrado foi mais elevado. As Regionais de Saúde de Guarapuava, Foz do Iguaçu, Cornélio Procópio e Ivaiporã tiveram coeficiente de detecção superior a 3 casos por 10 mil habitantes (Tabela 1, Figura 1).

Dos 1.932 casos de hanseníase em tratamento no Paraná, em 2005, os homens foram ligeira maioria (55,1\%) (Tabela 2). Esse padrão foi cons- tante em todas as Regionais de Saúde, exceto nas Regionais de Saúde de Foz do Iguaçu e de União da Vitória, nas quais, 55,3\% e 52,3\% eram do sexo feminino, respectivamente. A classificação operacional multibacilar predominou sobre a paucibacilar, com $64,7 \%$ do total dos casos diagnosticados (Tabela 2). A Regional de Foz do Iguaçu foi a que obteve maior diagnóstico de casos multibacilares (82,4\%), e a Regional de Cornélio Procópio, o maior percentual de casos paucibacilares (55\%). Dos casos de hanseníase diagnosticados quanto a sua forma clínica, prevaleceu a virchowiana, com $32,7 \%$ do total (Tabela 2). A Regional com maior número de casos virchowianos foi a de Ponta Grossa, com 59\%. Os resultados indicaram ainda que $41,3 \%$ dos pacientes diagnosticados, no Paraná, tinham mais de cinco lesões de pele.

O coeficiente de detecção da hanseníase, no Estado do Paraná, para a população de 0 a 14 anos, em 2005, foi de 0,17 caso por 10 mil habitantes. Nas Regionais de Saúde de Cianorte e Ivaiporã, foi de 1,13 e 1,22 caso por 10 mil habitantes, respectivamente, configurando-se uma situação hiperendêmica, conforme parâmetro do Ministério da Saúde. As Regionais de Saúde de 
Coeficiente de detecção e prevalência da hanseníase (por 10 mil habitantes) segundo Regional de Saúde. Paraná, Brasil 2005.

2a) Coeficiente de detecção

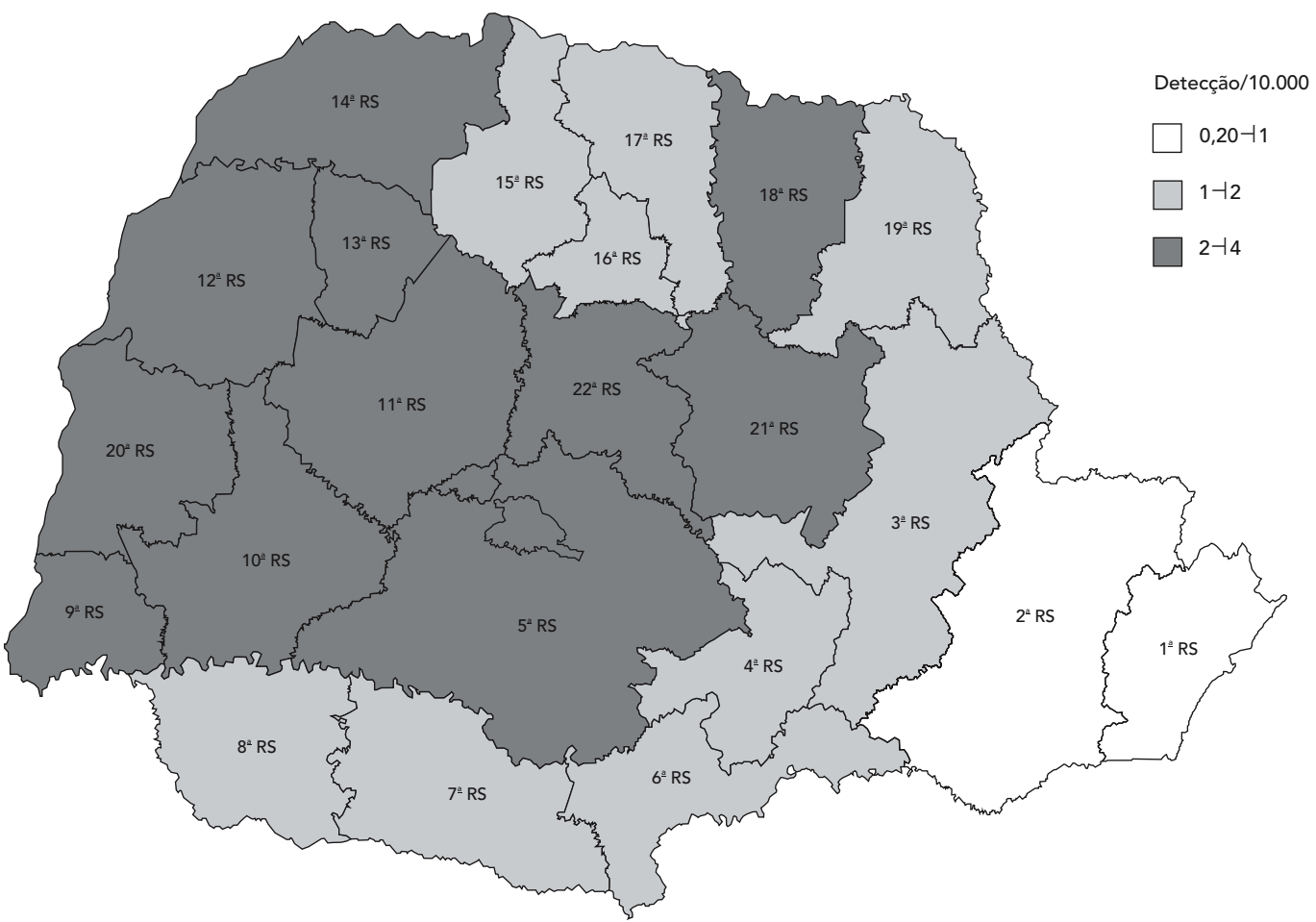

2b) Coeficiente de prevalência

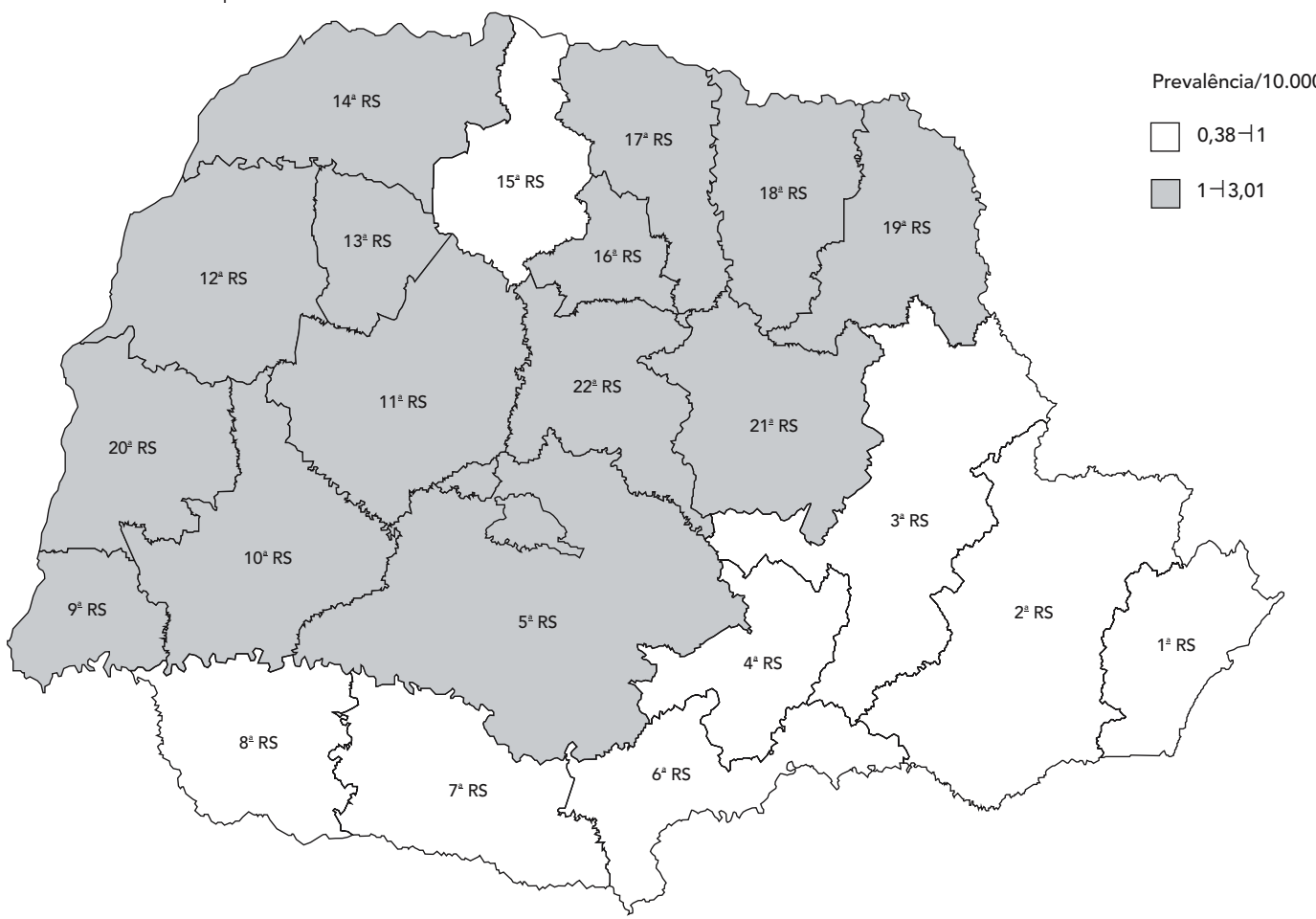

Fontes: Sistema de Informação de Agravos de Notificação (SINAN)/Secretaria Estadual de Saúde do Paraná, 2005; Tabnet

(Departamento de Informática do SUS, Ministério da Saúde, Brasil), 2005 
Foz do Iguaçu (0,58 caso por 10 mil habitantes), Telêmaco Borba $(0,57$ caso por 10 mil habitantes) e Paranavaí (0,56 caso por 10 mil habitantes) apresentaram parâmetro muito alto. As Regionais de Saúde de Guarapuava e Cascavel, ambas com coeficiente de detecção de 0,40 caso para cada 10 mil habitantes, enquadraram-se em padrão alto de endemicidade para menores de 15 anos (Figura 3).

\section{Discussão}

O comportamento do coeficiente de detecção da hanseníase no Estado do Paraná vem se mantendo quase que constante nos últimos seis anos. No Estado de São Paulo, o coeficiente de detecção manteve-se constante nos últimos trinta anos e, no período de 1991 a 2002, mostrou leve decréscimo ${ }^{16}$.

A OMS pontua que, no Brasil, a tendência da detecção da hanseníase não apresentou declínio nos últimos anos 2. Vijayakumaran et al. 17 afirmam que a detecção de casos novos não apresentou o declínio esperado nos países endêmicos. Os coeficientes de detecção continuam elevados e não foram alterados após quase dez anos de alta cobertura com a poliquimioterapia 18 .

Os coeficientes de detecção são indicadores de transmissibilidade da hanseníase ${ }^{19}$. No entanto, a redução desse indicador deve ser analisada com critério, porque a redução pode ser efeito da substituição da busca ativa de casos pela detecção passiva, possivelmente por falta de conscientização dos profissionais e da comunidade 18. É possível também que, em áreas onde a hanseníase esteja próxima de seu limiar de eliminação, a sensibilidade do serviço de saúde em detectar os casos novos fique comprometida devido à redução do aparecimento de casos suspeitos.

A Tabela 1 mostra que as Regionais de Saúde de Foz do Iguaçu, Guarapuava, Cornélio Procópio e Ivaiporã obtiveram os mais elevados coeficientes de detecção do período 2000 a 2005, muito superiores aos valores encontrados para o Estado do Paraná, indicando que as áreas geográficas possuem diferentes níveis de transmissibilidade da hanseníase, concordante com os relatos de que estatísticas globais sumarizadas em indicadores globais de morbidade podem esconder as variações regionais e de aglomerados locais 20,21, o que condiz com os relatos de Ranganadha et al. 22, haja vista que, no Distrito de Subarnapur, Estado de Orissa, Índia, nos anos de 2002 a 2004, houve uma queda súbita nos casos novos descobertos, contrariando a tendência da detecção em
Tabela 2

Inscritos no Programa de Controle da Hanseníase segundo sexo, forma clínica, classificação operacional e número de lesões de pele. Paraná, Brasil, 2005.

\begin{tabular}{lcc}
\hline & $\mathbf{N}$ & $\%$ * \\
\hline Sexo $(\mathrm{N}=1.932)$ & & \\
Masculino & 1.064 & 55,1 \\
Feminino & 868 & 44,9 \\
Forma clínica (N = 1.892) & & 17,3 \\
Indeterminada & 327 & 25,0 \\
Tuberculóide & 473 & 20,8 \\
Dimorfa & 394 & 32,7 \\
Virchowiana & 618 & 4,2 \\
Não classificada & 80 & 35,3 \\
Classificação operacional (N =1.907) & & 64,7 \\
Paucibacilar & 674 & \\
Multibacilar & 1.233 & 12,4 \\
Lesões de pele (N $=1.930)$ & & 19,7 \\
Nenhuma lesão & 240 & 26,5 \\
Lesão única & 381 & 41,4 \\
2-5 lesões & 511 & 798 \\
> 5 lesões & & \\
\hline
\end{tabular}

* Excluídos os ignorados.

Fonte: Sistema de Informação de Agravos de Notificação (SINAN)/Secretaria Estadual de Saúde do Paraná, 2005.

países endêmicos. É necessário, portanto, que se conheça a situação epidemiológica local para a definição de ações prioritárias conforme cada situação.

Portanto, calcular o coeficiente de detecção de uma região ou de aglomerados locais constitui-se em uma ferramenta necessária para identificar a realidade endêmica da doença. O conhecimento do coeficiente de detecção proporciona subsídio para que os serviços de saúde busquem estratégias para a realização de ações programáticas no processo de detecção precoce, tratamento e acompanhamento das pessoas e famílias atingidas pela hanseníase.

Os elevados coeficientes de detecção encontrados nas Regionais de Saúde de Foz do Iguaçu, Guarapuava, Cornélio Procópio e Ivaiporã sugerem que são áreas de detecção tardia, com alta transmissibilidade da hanseníase, merecendo especial atenção por parte dos gestores de saúde. Supõe-se, pelo comportamento do coeficiente de detecção, que essas Regionais de Saúde necessitarão de maior tempo para atingirem a meta de eliminação da hanseníase do que as demais Regionais de Saúde do Estado do Paraná.

O coeficiente de detecção, na população de 0 a 14 anos, em 2005, nas Regionais de Saúde de Foz 
Coeficiente de detecção da hanseníase na população menor de 15 anos (por 10 mil habitantes) segundo Regional de Saúde. Paraná, Brasil, 2005.

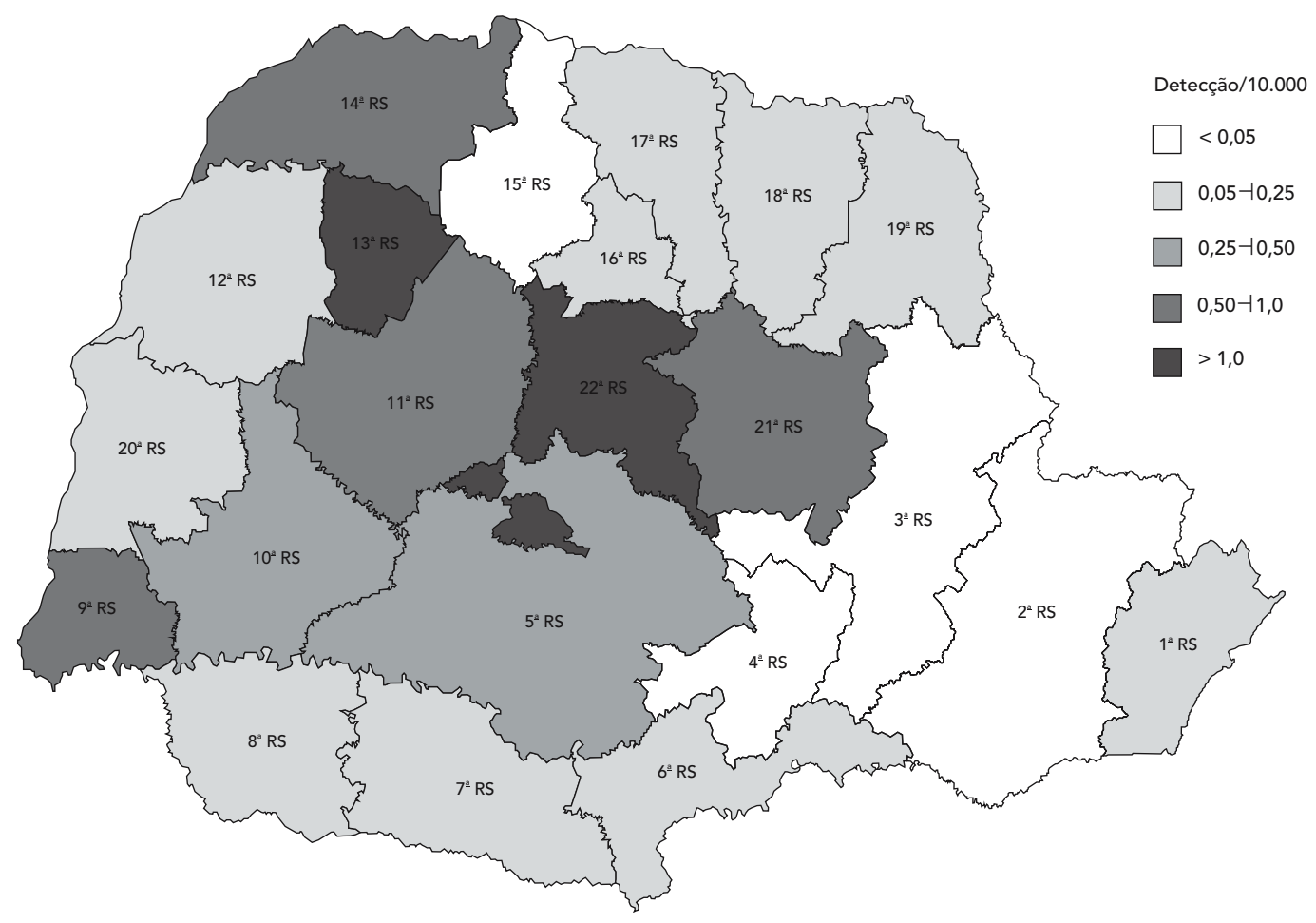

Fontes: Sistema de Informação de Agravos de Notificação (SINAN)/Secretaria Estadual de Saúde do Paraná, 2005

do Iguaçu, Telêmaco Borba, Paranavaí, Guarapuava e Cascavel, apresentou níveis endêmicos que variaram de alto a hiperendêmico. A detecção de casos em menores de 15 anos indica exposição precoce ao bacilo, possivelmente por existir importante transmissibilidade da doença e sugere também a presença intensa de casos bacilíferos entre as populações. A presença da doença em menores de 15 anos é utilizada como indicador do grau de transmissibilidade da hanseníase e também existe relação entre a proporção de casos em menores de 15 anos e a gravidade da endemia em uma localidade.

O panorama da eliminação da hanseníase, observado pelos altos coeficientes de detecção geral e em menores de 15 anos, sugere que, em algumas Regionais de Saúde, ainda existe grande número de doentes transmissores não diagnosticados, fato que pode estar dificultando a eliminação da doença no Paraná.

Discute-se ainda se as regiões que possuem menores coeficientes de detecção são aquelas onde estão esgotados os casos de hanseníase ou a sensibilidade em detectar casos novos foi diminuída. Há municípios no estado que estão em silêncio epidemiológico, isto é, não possuem casos diagnosticados, indicando que é nula a sensibilidade do serviço local de saúde na detecção da hanseníase. Considerando a distribuição da doença no estado, essa situação é tão preocupante quanto a daquelas localidades com alto coeficiente de detecção.

A prevalência da hanseníase, no Paraná, já apresentou níveis de hiperendemia. Atualmente, o coeficiente de prevalência possui parâmetro médio de endemicidade, em conformidade com os indicadores do Ministério da Saúde, embora o estado ainda apresente a maior prevalência da Região Sul do país.

A tendência da prevalência da hanseníase no Brasil é descendente e também foi observada no Estado do Paraná, embora, neste trabalho, a queda tenha sido muito leve, passando de 1,20 para 1,12 caso por 10 mil habitantes. A redução 
no tempo de tratamento que, anteriormente, era realizado em 24 meses para casos multibacilares e 12 meses para paucibacilares ou até mesmo pela vida toda, atualmente, é de 12 e 6 meses para multibacilares e paucibacilares, respectivamente. A diminuição no tempo de tratamento contribuiu decididamente para a redução da prevalência da doença em todo o mundo 12 .

Verificou-se que as Regionais de Saúde de Guarapuava, Foz do Iguaçu e Ivaiporã obtiveram os maiores coeficientes de prevalência no ano de 2005, indicando que, entre essas populações, provavelmente, exista intensa circulação de bacilos e focos antigos da doença. Contrariamente, oito Regionais de Saúde, em 2005, apresentaram coeficiente de prevalência menor do que 1 caso por 10 mil habitantes, concordante com os estudos de Norman et al. 23 em Gudiyatham Taluk, Índia, do Ministério da Saúde ${ }^{6}$, nos estados de Santa Catarina e Rio Grande do Sul e de Subramanian et al. 24 em Tamil Nadu, Índia, que são localidades que já atingiram a meta de eliminação proposta pela OMS.

Na Figura 1, percebe-se que as Regionais de Saúde com coeficientes de prevalência superiores a 1 caso por 10 mil habitantes são as mesmas com detecção elevada e mostra também as Regionais de Saúde Metropolitana e Paranaguá com coeficiente de prevalência e coeficiente de detecção baixos, conforme os parâmetros do Ministério da Saúde.

O coeficiente de detecção ultrapassando o de prevalência, como observado no Estado do Paraná, é uma tendência encontrada na maioria dos países endêmicos e está em concordância com o perfil esperado para as regiões em fase de eliminação da doença. Contudo, na Regional de Saúde de Paranaguá, o coeficiente de prevalência foi maior que o coeficiente de detecção, podendo ser resultado de questões operacionais, entre elas, o atraso no registro da cura do paciente no sistema de informação.

O coeficiente de prevalência baixo e o coeficiente de detecção elevado, muitas vezes ultrapassando o coeficiente de prevalência, conforme cenário de tendência secular brasileira 25 , são condizentes com uma prevalência influenciada pelo esquema terapêutico poliquimioterapia, o que corrobora os achados de Raja ${ }^{26}$, visto que a poliquimioterapia provocou um impacto sensível no coeficiente de prevalência da hanseníase em Andhra Pradesh, Índia.

O cenário da hanseníase observado pelo coeficiente de prevalência é sugestivo de que o Paraná está no limiar de eliminação, visto que o coeficiente apresentou parâmetro de endemicidade médio, em 2004 e 2005, e vem aproximando-se da meta de eliminação proposta pela OMS, que é de 1 ou menos de 1 caso de hanseníase para cada 10 mil habitantes.

As Regionais de Saúde de Ivaiporã, Foz do Iguaçu e Guarapuava obtiveram os maiores coeficientes de prevalência, variando de 2,14 a 3,01 casos por 10 mil habitantes, respectivamente, indicando que o trabalho está sendo executado, mas essas Regionais de Saúde precisam ampliar seus esforços para atingir a meta de eliminação. Por outro lado, em 2005, as Regionais de Saúde Paranaguá, Metropolitana, Ponta Grossa, Irati, União da Vitória, Pato Branco, Francisco Beltrão e Maringá alcançaram a meta de eliminação.

É importante ressaltar que, quanto ao uso do coeficiente de prevalência como indicador de eliminação da doença, ainda não existe consenso entre os autores. Khaitan et al. 27 ressaltam que é conflitante basear-se na prevalência para avaliar a redução da endemia, porque a própria OMS assevera que controlar uma doença é reduzir os coeficientes de incidência e prevalência e a morbidade a um nível localmente aceitável, com base nas características epidemiológicas da localidade como resultado de esforços propositais 28 .

Não obstante, no documento da OMS, Global Strategy for Further Reducing the Loprosy Burden and Sustaining Leprosy Control Activities, Plan Period: 2006-2010 [Estratégia Global para Aliviar a Carga da Hanseníase e Manter as Atividades de Controle] 28 , fica claro que uma das mudanças decisivas na organização do controle da hanseníase é o uso da detecção de casos como principal indicador de monitoramento, mas a prevalência ainda será o indicador cardeal até que a meta de eliminação seja atingida. Contudo, Andrade et al. 20 sugerem que o coeficiente de prevalência é uma ferramenta frágil para mensurar a demanda dos serviços de saúde e que o aumento do coeficiente de detecção, nos últimos anos, é resultado de variações da transmissão da hanseníase e mudanças operacionais do programa.

Verificou-se, para o ano de 2005, que os casos de hanseníase, no Paraná, foram maiores para os homens, embora, nas Regionais de Saúde de Foz do Iguaçu e União da Vitória, a porcentagem foi superior entre as mulheres, o que denota expansão da doença e deve ser utilizado como indicador epidemiológico para a elaboração de estratégias de vigilância. Ainda, a partir do número de casos multibacilares, pode-se afirmar que o Estado do Paraná possui grande porcentagem de casos diagnosticados nas formas graves da doença, e que a presença de bacilos circulantes é intensa, principalmente na área que abrange as Regionais de Saúde de Foz do Iguaçu, Ivaiporã, Ponta Grossa e Cascavel, onde o percentual de casos multibacilar é superior a $70 \%$. 
Quando os casos foram classificados quanto à forma clínica, observou-se que o Paraná é área com detecção tardia da hanseníase devido ao alto número de casos virchowianos. $\mathrm{Na} \mathrm{Re-}$ gional de Saúde de Foz do Iguaçu, prevaleceram os casos dimorfos e virchowianos, com $73,5 \%$ do total quando agrupados. No que diz respeito ao número de lesões de pele, é consenso, na literatura, que múltiplas lesões cutâneas significam estágios adiantados da doença, indicando que o caso é bacilífero, significa ainda diagnóstico tardio. Os casos diagnosticados mais tardiamente, por apresentarem múltiplas lesões de pele, foram observados nas Regionais Metropolitana e Foz do Iguaçu, com $25 \%$ e $18,7 \%$ dos casos, respectivamente.

O Instituto Lauro de Souza Lima, a pedido da Organização Pan-Americana da Saúde (OPAS) e da OMS, realizou investigação em vários estados do Brasil - inclusive no Paraná, selecionando os municípios de Toledo, Pitanga, Londrina e Curitiba - aplicando o exercício Monitoramento da Eliminação da Hanseníase (LEM) e encontrou maior quantidade de casos graves da doença, exemplificado maior detecção de casos multibacilares em relação aos paucibacilares, assegurando que a descoberta pode indicar endemia antiga que está sendo diagnosticada e tratada, distribuição epidemiológica característica do Brasil 29.

Sendo assim, com base no pressuposto anterior, os altos percentuais de casos multibacilares, a presença de localidades com maior proporção de casos entre as mulheres, o alto percentual de casos das formas clínicas virchowiana e dimorfa e predominância de casos com múltiplas lesões cutâneas indicam perfil de esgotamento da doença no estado, isto é, endemia em fase final, margeando para atingir os níveis estabelecidos na meta de eliminação da OMS.

Neste estudo, no Estado do Paraná, a queda da prevalência mediante a organização do sistema de informação, a implementação na capacidade de diagnóstico evidenciada pelo coeficiente de detecção e o aumento do número de casos novos podem ser resultado da ampliação da rede de diagnóstico oportunizado pela descentralização das atividades de controle da hanseníase - efeito da municipalização.

O plano de eliminação da hanseníase, no Brasil, está fundamentado estruturalmente em uma proposta de ampliação da rede de diagnóstico e atenção à pessoa atingida pela hanseníase, mediante a descentralização das atividades para os serviços de atenção básica e a implementação de equipes do Programa Saúde da Família (PSF). O que pode ter contribuído para um melhor acesso da população aos serviços de saúde e conseqüentemente melhoria na cobertura da demanda 30 .

A descentralização da assistência à hanseníase deve ser vista como um importante instrumento, porém não único, a ser utilizado como estratégia para eliminação da hanseníase. Fatores regionais culturais, sócio-econômicos, geográficos e políticos devem ser considerados para direcionar as decisões. Independente da eliminação da doença ocorrer no país até o prazo estabelecido, a endemia permanecerá por um tempo maior em microrregiões, em nível municipal 31 .

A perspectiva de eliminação da hanseníase no Estado do Paraná pode ser vista no contexto dos coeficientes de detecção geral, coeficiente de detecção em menores de 15 anos, coeficiente de prevalência e pelas características clínicas e operacionais dos casos. Tendo como base o coeficiente de prevalência que é o indicador de eliminação utilizado por todos os países membros da OMS, conclui-se que o Paraná encontra-se próximo de atingir a meta de eliminação estipulada, porque a prevalência pontual foi de 1,12 caso por 10 mil habitantes, e, em oito Regionais de Saúde, a meta já foi alcançada no ano de 2005. Contudo, o coeficiente de detecção que é o indicador de transmissibilidade da hanseníase não diminuiu durante os seis anos do estudo, mostrando que ainda existe uma importante circulação de bacilos e um número significativo de adoecimento por hanseníase no estado. Portanto, a redução do coeficiente de prevalência não ocorreu devido à diminuição do número de casos diagnosticados, mas, sim, pelo aumento do número de altas.

Os esforços do estado e das Regionais de Saúde devem ser mantidos e aprimorados principalmente naquelas regiões onde os coeficientes de detecção e de prevalência ainda persistem com parâmetros de endemicidade altos e médios e também porque os casos da doença continuarão a ocorrer em números apreciáveis, durante muitos anos, mesmo após o alcance da meta de eliminação. 


\section{Resumo}

A hanseníase, moléstia infecto-contagiosa endêmica, constitui ainda problema de saúde pública na maioria dos estados brasileiros, embora, nos últimos anos, venha apresentando queda importante em seu coeficiente de prevalência. Este estudo tem por objetivo analisar a perspectiva de eliminação da hanseníase no Estado do Paraná por meio dos coeficientes de detecção e de prevalência, nos anos de 2000 a 2005, além do sexo, idade, forma clínica, classificação operacional e número de lesões de pele. A fonte de informações foi o banco de dados do Sistema de Informação de Agravos de Notificação (SINAN) e o programa Tabnet. Os coeficientes de detecção e de prevalência da hanseníase no Paraná permaneceram sem alteração no período de estudo. Verificou-se, no Paraná, segundo as Regionais de Saúde, que o coeficiente de detecção da hanseníase foi, em sua maioria, alto ou muito alto. Quanto à prevalência, oito Regionais de Saúde alcançaram a meta de eliminação. Conclui-se que o Estado do Paraná está próximo de alcançar a meta de eliminação da hanseníase. Recomenda-se o fortalecimento das ações para que a eliminação da doença como um problema de saúde pública seja uma realidade.

Hanseníase; Notificação de Doenças; Prevalência

\section{Referências}

1. Lockwood DNJ. Leprosy elimination: a virtual phenomenon or a reality? BMJ 2002; 324:1516-8.

2. World Health Organization. Leprosy global situation. Wkly Epidemiol Rec 2005; 34:289-96.

3. Ho CK, Lo KK. Epidemiology of leprosy and response to treatment in Hong Kong. Hong Kong Med J 2006; 12:174-9.

4. Definición de caso: lepra. Bol Epidemiol (Wash) 2002; 23:15-6.

5. Brasil. Portaria GM no. 31 de 8 de julho de 2005 . Estabelece indicador epidemiológico para avaliação da prevalência da hanseníase. Diário Oficial da União 2005; 11 jul.

6. Meima A, Richardus JH, Habbema JDF. Trends in leprosy case detection worldwide since 1985. Lepr Rev 2004; 75:19-33.

7. Secretaria de Vigilância em Saúde, Ministério da Saúde. Meta do Ministério da Saúde em relação à hanseníase é de um caso por 10 mil habitantes até final de 2005. http://dtr2001.saude.gov.br/ svs/destaques/hanseniase.htm (acessado em 14/ Nov/2005)

8. Ignotti E. O paradoxo dos indicadores de monitoramento da eliminação da hanseníase [Tese de Doutorado]. Rio de Janeiro: Escola Nacional de Saúde Pública, Fundação Oswaldo Cruz; 2004.

\section{Colaboradores}

Os autores participaram na concepção e planejamento da pesquisa. R. A. Silva Sobrinho participou na busca e análise de dados e redação do manuscrito. T. A. F. Mathias participou na orientação e redação do manuscrito.
9. Meima A, Gupte MD, van Orrtmarssen GJ, Habbema JD. Trends in leprosy case detection rates. Int J Lepr Other Mycobact Dis 1997; 65:30519.

10. Secretaria de Estado de Saúde do Paraná. Estratégias de eliminação da hanseníase no Estado do Paraná. Curitiba: Instituto de Saúde do Paraná; 2005.

11. Sharma R. Complete elimination of leprosy still elusive. BMJ 2001; 322:318.

12. Lockwood DNJ, Suneetha S. Leprosy: too complex a disease for a simple elimination paradigm. Bull World Health Organ 2005; 83:230-5.

13. Martelli CMT, Stefani MMA, Penna GO, Andrade ALSS. Endemias e epidemias brasileiras, desafios e perspectivas de investigação científica: hanseníase. Rev Bras Epidemiol 2002; 5:273-85.

14. Ministério da Saúde. Informações e análise de situação de saúde. http://portal.saude.gov.br/ portal/svs/area.cfm?id_area $=460$ (acessado em 20/Set/2006).

15. Departamento de Atenção Básica, Secretaria de Políticas de Saúde, Ministério da Saúde. Hanseníase atividades de controle e manual de procedimentos. Brasília: Ministério da Saúde; 2004. 
16. Opromolla PA, Dalben I, Cardim M. Análise da distribuição espacial da hanseníase no Estado de São Paulo, 1991-2002. Rev Bras Epidemiol 2005; 8:35664.

17. Vijayakumaran P, Prasad B, Krishnamurthy P. Trends in new case-detection leprosy in Bihar, India. Indian J Dermatol Venereol Leprol 2006; 78:145-51.

18. Gomes CCD, Gonçalves HS, Pontes MAA, Penna GO. Perfil clínico-epidemiológico dos pacientes diagnosticados com hanseníase em um centro de referência na Região Nordeste do Brasil. An Bras Dermatol 2005; 80(3 Suppl):283-8.

19. International Federation of Anti-Leprosy Associations. The interpretation on epidemiological indicators in leprosy. London: International Federation of Anti-Leprosy Associations; 2001. (Technical Bulletin).

20. Andrade VLG, Sabroza PC, Araújo AJG. Fatores associados ao domicílio e à família na determinação da hanseníase, Rio de Janeiro, Brasil. Cad Saúde Pública 1994; 10 Suppl 2:S281-92.

21. Martelli CMT, Morais Neto OL, Andrade ALSS, Silva SA, Silva IM, Zicker F. Spatial patterns of leprosy in an urban area of central Brazil. Bull World Health Organ 1995; 73:315-9.

22. Ranganadha RPV, Peri S, Porichha D, Nehemaiah E. A review of trends in new case-detection in Subarnapur district of Orissa. Indian J Dermatol Venereol Leprol 2006; 78:153-65.

23. Norman G, Raja S, Bhushanam JD, Samuel P. Trends in leprosy over fifty years in Gudiyatham Taluk, Vellore, Tamil Nadu. Indian J Dermatol Venereol Leprol 2006; 78:167-85.

24. Subramanian M, Thorat DM, Krishnan CB, Baig AA, Prabakaran I, Hassan TF, et al. Epidemiological trends of leprosy elimination in CLTRI rural field operation area Tamil Nadu, India. Indian J Dermatol Venereol Leprol 2006; 78:203-14.
25. Lechat MF. Rationale for the preventive treatment for leprosy. Int J Lepr Other Mycobact Dis 1999; 67:63-6.

26. Raja RB. New case-detection trends in a multi-drug therapy programme: definite reduction in sight. Indian J Dermatol Venereol Leprol 2006; 78:12336.

27. Khaitan BK, Tejasvi T, Aggarwal K, Khanna N. Final push of leprosy: it is prudent to pause before declaration! Indian J Dermatol Venereol Leprol 2006; 72:151-3.

28. World Health Organization. Global strategy for further reducing the leprosy burden and sustaining leprosy control activities, plan period: 2006-2010. http://www.who.int/lep/Reports/GlobalStrategyPDF-verison.pdf (acessado em 14/Ago/2005).

29. Organização Mundial da Saúde. Monitoramento da eliminação da hanseníase: LEM: manual para monitores. http://www.opas.org.br/prevencao/ site/UploadArq/manualLEM.pdf (acessado em 03/Mar/2006).

30. Lapa TM, Albuquerque MFPM, Carvalho MS, Silveira Júnior JC. Análise da demanda de casos de hanseníase aos serviços de saúde através do uso de técnicas de análise espacial. Cad Saúde Pública 2006; 22:2575-83.

31. Cunha MD, Cavaliere FAM, Hércules FM, Duraes SMB, Oliveira MLW, Matos HJ. Os indicadores da hanseníase e as estratégias de eliminação da doença, em município endêmico do Estado do Rio de Janeiro, Brasil. Cad Saúde Pública 2007; 23:1187-97.

Recebido em 17/Mar/2007

Versão final reapresentada em 26/Jun/2007

Aprovado em 13/Jul/2007 